УДК 517.958:532.516

\title{
Systematization and Analysis of Integrals of Motion for an Incompressible Fluid Flow
}

\author{
Alexander V. Koptev* \\ Admiral Makarov State University of Maritime and Inland Shipping \\ Dvinskaya, 5/7, Saint-Petersburg, 198035
}

Russia

Received 7.05.2017, received in revised form 30.09.2017, accepted 20.02.2018

An analysis of integrals of motion of an incompressible fluid flow both known and new obtained by author are presented in the paper. It was found that the known integrals of Lagrange-Cauchy, Bernoulli and Euler-Bernoulli are special cases of a new more general integral. It was shown that the set of all integrals of motion of an incompressible fluid form a logical chain which can be represented as a tree.

Keywords: incompressible fluid, motion, Navier-Stokes equations, Euler equations, partial derivative, root integral, stream pseudo-function, potential, tree.

DOI: 10.17516/1997-1397-2018-11-3-370-382.

\section{Introduction}

Model of a viscous incompressible fluid is one of the most frequently used in fluid mechanics. For this case the density and all other physical characteristics of the fluid are constant, and the major unknowns are the components of the velocity vector $u, v, w$ and pressure $p$. Generally accepted equations to describe motion of such a media are the Navier-Stokes equations [1-4]. They can be represented in dimensionless form as

$$
\begin{gathered}
\frac{\partial u}{\partial t}+u \frac{\partial u}{\partial x}+v \frac{\partial u}{\partial y}+w \frac{\partial u}{\partial z}=-\frac{\partial(p+\Phi)}{\partial x}+\frac{1}{R e} \Delta u \\
\frac{\partial v}{\partial t}+u \frac{\partial v}{\partial x}+v \frac{\partial v}{\partial y}+w \frac{\partial v}{\partial z}=-\frac{\partial(p+\Phi)}{\partial y}+\frac{1}{R e} \Delta v \\
\frac{\partial w}{\partial t}+u \frac{\partial w}{\partial x}+v \frac{\partial w}{\partial y}+w \frac{\partial w}{\partial z}=-\frac{\partial(p+\Phi)}{\partial z}+\frac{1}{R e} \Delta w \\
\frac{\partial u}{\partial x}+\frac{\partial v}{\partial y}+\frac{\partial w}{\partial z}=0
\end{gathered}
$$

Here $\Delta$ is a three-dimensional Laplace operator: $\Delta=\frac{\partial^{2}}{\partial x^{2}}+\frac{\partial^{2}}{\partial y^{2}}+\frac{\partial^{2}}{\partial z^{2}}, \Phi$ is the potential of external forces, Re is the Reynolds number.

The vanishing of the kinematic viscosity corresponds to $R e=+\infty$. For this case the righthand sides of (1-3) are significantly simplified. Terms proportional to $\frac{1}{R e}$ are neglected, and the Navier-Stokes equations turn into Euler equations. The term "ideal" or "perfect" fluid are usually used for such a medium.

*Alex.Koptev@mail.ru

(c) Siberian Federal University. All rights reserved 
The Navier - Stokes and Euler equations have numerous applications to practical problems. Their complex study is one of the areas of modern mathematical physics [5-6]. Today, however, many issues are not fully clarified and they require further study. One of the main problems is the lack of a constructive method of solving these equations. An important step in this direction is the construction of integrals of motion. The provisions of classical fluid mechanics in this regard are as follows. There are three integrals that are convenient for practical application [1-4].

The Lagrange-Cauchy integral is for potential non-stationary fluid flow. For an incompressible medium it can be represented in dimensionless variables as

$$
p+\Phi+\frac{U^{2}}{2}+\frac{\partial \varphi}{\partial t}=f(t)
$$

where $U=\sqrt{u^{2}+v^{2}+w^{2}}, \varphi$ is the velocity potential, $f(t)$ is an arbitrary function of time.

The Lagrange-Cauchy integral is just for the potential motion of both ideal and viscous media.

The Bernoulli integral is for the steady-state motion along the stream line of an ideal medum. In the case of an incompressible medium it can be written in dimensionless variables as

$$
p+\Phi+\frac{U^{2}}{2}=C_{s l}
$$

where $C_{s l}$ denotes a constant that depends on the choice of a stream line. The subscript "sl" from English "stream line" is underlines this pattern.

The constant $C_{s l}$ on the right-hand side (6) does not depend on the choice of a stream line if the additional condition

$$
\vec{U} \times \vec{\Omega}=0
$$

is hold, where $\vec{U}$ is the velocity vector, $\vec{\Omega}$ is the swirl vector, " $\times$ " means the vector product.

In this case the integral is often called the Euler-Bernoulli integral. It can be written as

$$
p+\Phi+\frac{U^{2}}{2}=C
$$

where $C$ denotes an absolute constant that does not depend on the choice of a stream line.

These integrals are the basic relations of classical fluid mechanics. A wide variety of problems were solved on the basis of these integrals. But even for these well known relations not all questions are clarified adequately. For example, it is not clear how the Bernoulli integral (6) is transformed if the characteristic point is not taken along a stream line. Then relation (6) fails. It is also not clear what we will have instead of (5) if we abandon the condition of potentiality of motion.

Assumptions and range of applicability for integrals (5), (6), (8) are different. But each of them contains the same combination $p+\Phi+\frac{U^{2}}{2}$ which is usually called the Bernoulli trinomial. We face the question on the existence of a general root integral which also would contain the Bernoulli trinomial and include relations (5), (6), (8) as special cases.

\section{Root integral}

The procedure to construct general root integral was proposed [7-9]. This procedure is based on the representation of equations (1-4) in free divergence form

$$
\frac{\partial P_{i}}{\partial x}+\frac{\partial Q_{i}}{\partial y}+\frac{\partial R_{i}}{\partial z}+\frac{\partial S_{i}}{\partial t}=0
$$


where $P_{i}, Q_{i}, R_{i}, S_{i}$ are some combinations of unknowns and their first derivatives with respect to spatial coordinates.

Each equation of the form (9) admits integration. The resulting relation can be combined and simplified by full or partial exclusion of the non-divergent terms. Some of the resulting relations can be brought to the form (9) and then integrated again. Transformations result in nine basic relations. Using the simplest notation, they can be represented as

$$
\begin{aligned}
& p+\Phi+\frac{U^{2}}{2}+d+d_{t}=\alpha_{4}+\beta_{4}+\gamma_{4}, \\
& u^{2}-v^{2}+\frac{2}{R e}\left(-\frac{\partial u}{\partial x}+\frac{\partial v}{\partial y}\right)=-\frac{\partial^{2} \Psi_{10}}{\partial x^{2}}+\frac{\partial^{2} \Psi_{10}}{\partial y^{2}}-\frac{\partial^{2} \Psi_{11}}{\partial z^{2}}-\frac{\partial^{2} \Psi_{12}}{\partial z^{2}}+\frac{\partial^{2} \Psi_{15}}{\partial y \partial z}+ \\
& +\frac{\partial^{2} \Psi_{14}}{\partial x \partial z}+\frac{\partial}{\partial t}\left(-\frac{\partial \Psi_{1}}{\partial x}+\frac{\partial \Psi_{3}}{\partial y}+\frac{\partial\left(\Psi_{5}+\Psi_{6}\right)}{\partial z}\right)+3\left(\alpha_{4}-\beta_{4}\right), \\
& v^{2}-w^{2}+\frac{2}{R e}\left(-\frac{\partial v}{\partial y}+\frac{\partial w}{\partial z}\right)=\frac{\partial^{2} \Psi_{10}}{\partial x^{2}}+\frac{\partial^{2} \Psi_{11}}{\partial x^{2}}-\frac{\partial^{2} \Psi_{12}}{\partial y^{2}}+\frac{\partial^{2} \Psi_{12}}{\partial z^{2}}-\frac{\partial^{2} \Psi_{13}}{\partial x \partial y}- \\
& -\frac{\partial^{2} \Psi_{14}}{\partial x \partial z}+\frac{\partial}{\partial t}\left(\frac{\partial\left(\Psi_{1}+\Psi_{21}\right)}{\partial x}+\frac{\partial \Psi_{4}}{\partial y}-\frac{\partial \Psi_{6}}{\partial z}\right)+3\left(\beta_{4}-\gamma_{4}\right), \\
& u v-\frac{1}{R e}\left(\frac{\partial v}{\partial x}+\frac{\partial u}{\partial y}\right)=-\frac{\partial^{2} \Psi_{10}}{\partial x \partial y}+\frac{1}{2} \frac{\partial}{\partial z}\left(-\frac{\partial \Psi_{15}}{\partial x}+\frac{\partial \Psi_{14}}{\partial y}+\frac{\partial \Psi_{13}}{\partial z}\right)+ \\
& +\frac{1}{2} \frac{\partial}{\partial t}\left(-\frac{\partial \Psi_{3}}{\partial x}-\frac{\partial \Psi_{1}}{\partial y}-\frac{\partial\left(\Psi_{8}+\Psi_{9}\right)}{\partial z}\right)+\frac{1}{2}\left(\frac{\partial \alpha_{1}}{\partial z}-\frac{\partial \alpha_{3}}{\partial t}+\frac{\partial \beta_{1}}{\partial z}-\frac{\partial \beta_{2}}{\partial t}\right), \\
& u w-\frac{1}{R e}\left(\frac{\partial w}{\partial x}+\frac{\partial u}{\partial z}\right)=\frac{\partial^{2} \Psi_{11}}{\partial x \partial z}+\frac{1}{2} \frac{\partial}{\partial y}\left(-\frac{\partial \Psi_{15}}{\partial x}-\frac{\partial \Psi_{14}}{\partial y}-\frac{\partial \Psi_{13}}{\partial z}\right)+ \\
& +\frac{1}{2} \frac{\partial}{\partial t}\left(-\frac{\partial \Psi_{5}}{\partial x}+\frac{\partial\left(\Psi_{9}-\Psi_{7}\right)}{\partial y}+\frac{\partial \Psi_{2}}{\partial z}\right)-\frac{1}{2}\left(\frac{\partial \alpha_{1}}{\partial y}+\frac{\partial \alpha_{2}}{\partial t}-\frac{\partial \gamma_{1}}{\partial y}+\frac{\partial \gamma_{3}}{\partial t}\right), \\
& v w-\frac{1}{R e}\left(\frac{\partial w}{\partial y}+\frac{\partial v}{\partial z}\right)=-\frac{\partial^{2} \Psi_{12}}{\partial y \partial z}+\frac{1}{2} \frac{\partial}{\partial x}\left(\frac{\partial \Psi_{14}}{\partial y}+\frac{\partial \Psi_{15}}{\partial x}-\frac{\partial \Psi_{13}}{\partial z}\right)+ \\
& +\frac{1}{2} \frac{\partial}{\partial t}\left(\frac{\left(\partial \Psi_{7}+\Psi_{8}\right)}{\partial x}+\frac{\partial \Psi_{6}}{\partial y}+\frac{c \Psi_{4}}{\partial z}\right)-\frac{1}{2}\left(\frac{\partial \beta_{1}}{\partial x}+\frac{\partial \beta_{3}}{\partial t}+\frac{\partial \gamma_{1}}{\partial x}+\frac{\partial \gamma_{2}}{\partial t}\right), \\
& u=\frac{1}{2}\left(\frac{\partial}{\partial y}\left(-\frac{\partial \Psi_{3}}{\partial x}+\frac{\partial \Psi_{1}}{\partial y}+\frac{\partial \Psi_{7}}{\partial z}\right)+\frac{\partial}{\partial z}\left(-\frac{\partial \Psi_{5}}{\partial x}+\frac{\partial \Psi_{8}}{\partial y}-\frac{\partial \Psi_{2}}{\partial z}\right)\right)+ \\
& +\frac{1}{2}\left(\frac{\partial \alpha_{2}}{\partial z}+\frac{\partial \alpha_{3}}{\partial y}+\frac{\partial \delta_{1}}{\partial y}+\frac{\partial \delta_{2}}{\partial z}\right) \\
& v=\frac{1}{2}\left(\frac{\partial}{\partial x}\left(\frac{\partial \Psi_{3}}{\partial x}-\frac{\partial \Psi_{1}}{\partial y}-\frac{\partial \Psi_{7}}{\partial z}\right)+\frac{\partial}{\partial z}+\left(\frac{\partial \Psi_{9}}{\partial x}+\frac{\partial \Psi_{6}}{\partial y}-\frac{\partial \Psi_{4}}{\partial z}\right)\right)+ \\
& +\frac{1}{2}\left(\frac{\partial \beta_{2}}{\partial x}+\frac{\partial \beta_{3}}{\partial z}-\frac{\partial \delta_{1}}{\partial x}+\frac{\partial \delta_{3}}{\partial z}\right)
\end{aligned}
$$




$$
\begin{aligned}
w=\frac{1}{2}\left(\frac{\partial}{\partial x}\left(\frac{\partial \Psi_{5}}{\partial x}-\frac{\partial \Psi_{8}}{\partial y}+\frac{\partial \Psi_{2}}{\partial z}\right)+\frac{\partial}{\partial y}\left(-\frac{\partial \Psi_{9}}{\partial x}-\right.\right. & \left.\left.\frac{\partial \Psi_{6}}{\partial y}+\frac{\partial \Psi_{4}}{\partial z}\right)\right)+ \\
& +\frac{1}{2}\left(\frac{\partial \gamma_{2}}{\partial y}+\frac{\partial \gamma_{3}}{\partial x}-\frac{\partial \delta_{2}}{\partial x}-\frac{\partial \delta_{3}}{\partial y}\right) .
\end{aligned}
$$

Let us note that new values $\Psi_{i}$ appear in relations (10-18). Functions $\Psi_{i}$ are associated unknowns. They arise as a result of integration of original equations (1-4), and supplement the system unknowns. They are called "stream pseudo-functions" [9-11].

Functions $\alpha_{k}, \beta_{k}, \gamma_{k}, \delta_{k}$ are arbitrary functions of three variables, and they occur during the process of integration. These functions and their first derivatives are additives in the right-hand side of equations (10-18). Each of functions $\alpha_{k}, \beta_{k}, \gamma_{k}, \delta_{k}$ does not depend on one of variables $x, y, z$ and $t$, respectively. Then the following equalities must hold

$$
\frac{\partial \alpha_{k}}{\partial x}=\frac{\partial \beta_{k}}{\partial y}=\frac{\partial \gamma_{k}}{\partial z}=\frac{\partial \delta_{k}}{\partial t}=0
$$

Variables $d$ and $d_{t}$ are additives in relation (10). They represent the dissipative terms, and correspond to the reduced stationary and non-stationary dissipation, respectively. These variables are defined as follows

$$
\begin{gathered}
d=-\frac{U^{2}}{6}-\frac{1}{3}\left(\Delta_{x y} \Psi_{10}-\Delta_{x z} \Psi_{11}+\Delta_{y z} \Psi_{12}+\frac{\partial^{2} \Psi_{13}}{\partial x \partial y}-\frac{\partial^{2} \Psi_{14}}{\partial x \partial z}+\frac{\partial^{2} \Psi_{15}}{\partial y \partial z}\right), \\
d_{t}=-\frac{1}{3} \frac{\partial}{\partial t}\left(\frac{\partial\left(\Psi_{2}-\Psi_{1}\right)}{\partial x}+\frac{\partial\left(\Psi_{4}-\Psi_{3}\right)}{\partial y}+\frac{\partial\left(\Psi_{6}-\Psi_{5}\right)}{\partial z}\right) .
\end{gathered}
$$

Characters $\Delta_{y z}, \Delta_{x z}, \Delta_{x y}$ denote the partial Laplace operators with espect to spatial coordinates

$$
\Delta_{y z}=\frac{\partial^{2}}{\partial y^{2}}+\frac{\partial^{2}}{\partial z^{2}}, \quad \Delta_{x z}=\frac{\partial^{2}}{\partial x^{2}}+\frac{\partial^{2}}{\partial z^{2}}, \quad \Delta_{x y}=\frac{\partial^{2}}{\partial x^{2}}+\frac{\partial^{2}}{\partial y^{2}} .
$$

Relations (10-18) connect the major unknowns $u, v, w, p$, associated unknowns $\Psi_{i}$, and an arbitrary functions $\alpha_{k}, \beta_{k}, \gamma_{k}, \delta_{k}$. The order of derivatives of major unknowns is one that is less than their order in the original equations (1-4).

Relations (10-18) considered together represent the root integral of Navier-Stokes equations (1-4). The proof of this statement can be found in [9-10].

It is not surprising thing that the integral is represented by nine interdependent relations rather than one relation. If we do not introduce restrictions, such as potentiality of motion, ideality of medium, motion along a stream line then the number of relations are increased and their structure becomes more complex.

The presence of new associated unknown $\Psi_{i}$ in relations (10-18) is not surprising. Analogues of associated unknown are also present in integrals (5) and (6). The role of an associate unknown in integral (5) plays the velocity potential $\varphi$. The associated unknown in integral (6) is the value of the right-hand side $C_{s l}$ since this value depends on the stream line and therefore it is a function. An arbitrary additive function is also in integral (5). This is a function of time $f(t)$.

Let us note that the analysis of equations of motion of an incompressible fluid is essentially similar to that used earlier [7]. However, the consideration was limited to the case of $2 D$ equations and the final relation for integral has not been presented. Similar approach was used in [8]. In that paper the equation containing an associated unknown called "parametrically defined" differential equation.

The root integral of $3 D$ Navier - Stokes equations (1-4) is represented by nine interconnected relations (10-18). Comparison of these relations with known Lagrange-Cauchy (5), Bernoulli 
(6) and Euler-Bernoulli (8) integrals leads to the following statements. Each of integrals (5), (6), (8) is a special case of $(10-18)$.

\section{Integral of Lagrange-Cauchy as a special case of the root integral}

Let us consider the Lagrange-Cauchy integral. The following theorem is valid.

Theorem 2.1 The Lagrange-Cauchy (5) integral is a special case of the root integral (10-18).

Proof. Consider relations (10-18) and suppose that fluid flow is potential. Then the potential of velocity $\varphi$ exists and it satisfies the Laplace equation $\Delta \varphi=0$. The following equality holds $[1-4]$

$$
u=\frac{\partial \varphi}{\partial x}, v=\frac{\partial \varphi}{\partial y}, w=\frac{\partial \varphi}{\partial z}
$$

Each of velocity components also satisfies the Laplace equation

$$
\Delta u=0, \quad \Delta v=0, \quad \Delta w=0 .
$$

In this case the motion is irrotational so $\vec{\Omega}=0$. The latter is equivalent to three scalar equations

$$
\frac{\partial w}{\partial y}-\frac{\partial v}{\partial z}=0, \quad \frac{\partial u}{\partial z}-\frac{\partial w}{\partial x}=0, \quad \frac{\partial v}{\partial x}-\frac{\partial u}{\partial y}=0 .
$$

Using conditions (22-24), we have to prove that from (10-18) follows (5).

Relation (10) contains the largest number of unknowns and pressure $p$ appears only in this relation. The left-hand side of (10) differs by $d+d_{t}$ from Bernoulli trinomial. We take first derivatives of $d+d_{t}$ with respect to spatial coordinates and obtain three groups of equations.

2.1.1. Taking into account $(20),(21)$, we obtain derivative with respect to $\partial x$

$$
\begin{array}{r}
\frac{\partial\left(d+d_{t}\right)}{\partial x}=-\frac{1}{3}\left(u \frac{\partial u}{\partial x}+v \frac{\partial v}{\partial x}+w \frac{\partial w}{\partial x}\right)+\frac{1}{3}\left(-\frac{\partial^{3} \Psi_{10}}{\partial x^{3}}-\frac{\partial^{3} \Psi_{10}}{\partial x \partial y^{2}}+\frac{\partial^{3} \Psi_{11}}{\partial x^{3}}+\frac{\partial^{3} \Psi_{11}}{\partial x \partial z^{2}}-\right. \\
\left.-\frac{\partial^{3} \Psi_{12}}{\partial x \partial y^{2}}-\frac{\partial^{3} \Psi_{12}}{\partial x \partial z^{2}}-\frac{\partial^{3} \Psi_{13}}{\partial x^{2} \partial y}+\frac{\partial^{3} \Psi_{14}}{\partial x^{2} \partial z}-\frac{\partial^{3} \Psi_{15}}{\partial x \partial y \partial z}\right)+\frac{1}{3} \frac{\partial}{\partial t}\left(\frac{\partial^{2} \Psi_{2}}{\partial x^{2}}-\frac{\partial^{2} \Psi_{1}}{\partial x^{2}}+\frac{\partial^{2} \Psi_{4}}{\partial x \partial y}-\right. \\
\left.-\frac{\partial^{2} \Psi_{3}}{\partial x \partial y}+\frac{\partial^{2} \Psi_{6}}{\partial x \partial z}-\frac{\partial^{2} \Psi_{5}}{\partial x \partial z}\right)
\end{array}
$$

The second term of right-hand side contains third derivatives with respect to spatial coordinates. Let us transform this term with the use of relations (11-18). We find $\frac{2}{3} \frac{\partial}{\partial x}$ of (11), $\frac{1}{3} \frac{\partial}{\partial x}$ of (12), $\frac{\partial}{\partial y}$ of (13) and $\frac{\partial}{\partial z}$ of (14). Combining the above results, we obtain an expression for the term in the form

$$
\begin{aligned}
& \frac{1}{3}\left(\frac{\partial^{3} \Psi_{10}}{\partial x^{3}}-\frac{\partial^{3} \Psi_{10}}{\partial x \partial y^{2}}+\frac{\partial^{3} \Psi_{11}}{\partial x^{3}}+\frac{\partial^{3} \Psi_{11}}{\partial x \partial z^{2}}-\frac{\partial^{3} \Psi_{12}}{\partial x \partial y^{2}}-\frac{\partial^{3} \Psi_{12}}{\partial x \partial z^{2}}-\frac{\partial^{3} \Psi_{13}}{\partial x^{2} \partial y}+\frac{\partial^{3} \Psi_{14}}{\partial x^{2} \partial z}-\frac{\partial^{3} \Psi_{15}}{\partial x \partial y \partial z}\right)= \\
= & \frac{4}{3} u \frac{\partial u}{\partial x}-\frac{2}{3} v \frac{\partial v}{\partial x}-\frac{2}{3} w \frac{\partial w}{\partial x}+v \frac{\partial u}{\partial y}+u \frac{\partial v}{\partial y}+w \frac{\partial u}{\partial z}+u \frac{\partial w}{\partial z}+\frac{1}{R e}\left(-\frac{4}{3} \frac{\partial^{2} u}{\partial x^{2}}-\frac{1}{3} \frac{\partial^{2} v}{\partial x \partial y}-\frac{1}{3} \frac{\partial^{2} w}{\partial x \partial z}-\right.
\end{aligned}
$$




$$
\begin{aligned}
\left.-\frac{\partial^{2} u}{\partial y^{2}}-\frac{\partial^{2} u}{\partial z^{2}}\right)+ & \frac{\partial}{\partial t}\left(\frac{1}{3} \frac{\partial^{2} \Psi_{1}}{\partial x^{2}}+\frac{1}{2} \frac{\partial^{2} \Psi_{1}}{\partial y^{2}}-\frac{1}{3} \frac{\partial^{2} \Psi_{2}}{\partial x^{2}}-\frac{1}{2} \frac{\partial^{2} \Psi_{2}}{\partial z^{2}}-\frac{1}{6} \frac{\partial^{2} \Psi_{3}}{\partial x \partial y}-\frac{1}{3} \frac{\partial^{2} \Psi_{4}}{\partial x \partial y}-\frac{1}{6} \frac{\partial^{2} \Psi_{5}}{\partial x \partial z}-\right. \\
& \left.-\frac{1}{6} \frac{\partial^{2} \Psi_{6}}{\partial x \partial z}+\frac{1}{2} \frac{\partial^{2} \Psi_{7}}{\partial y \partial z}+\frac{1}{2} \frac{\partial^{2} \Psi_{8}}{\partial y \partial z}\right)+\frac{1}{2} \frac{\partial^{2} \alpha_{2}}{\partial z \partial t}+\frac{1}{2} \frac{\partial^{2} \alpha_{3}}{\partial y \partial t}+\frac{\partial \beta_{4}}{\partial x}+\frac{\partial \gamma_{4}}{\partial x} .
\end{aligned}
$$

Taking into account (26), we trasform (25) as

$$
\begin{gathered}
\frac{\partial\left(d+d_{t}\right)}{\partial x}=\frac{1}{R e}\left(-\Delta u-\frac{1}{3} \frac{\partial}{\partial x}\left(\frac{\partial u}{\partial x}+\frac{\partial v}{\partial y}+\frac{\partial w}{\partial z}\right)\right)+u\left(\frac{\partial u}{\partial x}+\frac{\partial v}{\partial y}+\frac{\partial w}{\partial z}\right)+v\left(\frac{\partial u}{\partial y}-\frac{\partial v}{\partial x}\right)+ \\
+w\left(\frac{\partial u}{\partial z}-\frac{\partial w}{\partial x}\right)+\frac{1}{2} \frac{\partial}{\partial t}\left(\frac{\partial^{2} \Psi_{1}}{\partial y^{2}}-\frac{\partial^{2} \Psi_{2}}{\partial z^{2}}-\frac{\partial^{2} \Psi_{3}}{\partial x \partial y}-\frac{\partial^{2} \Psi_{5}}{\partial x \partial z}+\frac{\partial^{2} \Psi_{7}}{\partial y \partial z}+\frac{\partial^{2} \Psi_{8}}{\partial y \partial z}\right)+ \\
+\frac{1}{2} \frac{\partial^{2} \alpha_{2}}{\partial z \partial t}+\frac{1}{2} \frac{\partial^{2} \alpha_{3}}{\partial y \partial t}+\frac{\partial \beta_{4}}{\partial x}+\frac{\partial \gamma_{4}}{\partial x} .
\end{gathered}
$$

Relation (27) can be significantly simplified. It follows from (23) that $\Delta u=0$. Taking into account continuity equation (4) we obtain that group of terms proportional to $\frac{1}{R e}$ vanishes.

The second group on right-hand side of (27) also vanishes due to (4) and (24). Let us use equation (16) and relation $\frac{\partial \delta_{k}}{\partial t}=0$ from (19). Then we obtain that all the remaining terms on the right-hand side of (27) are reduced to $\frac{\partial u}{\partial t}+\frac{\partial \beta_{4}}{\partial x}+\frac{\partial \gamma_{4}}{\partial x}$.

Then relation (27) takes the form

$$
\frac{\partial\left(d+d_{t}\right)}{\partial x}=\frac{\partial u}{\partial t}+\frac{\partial \beta_{4}}{\partial x}+\frac{\partial \gamma_{4}}{\partial x}
$$

2.1.2. Let us take the first derivative of $d+d_{t}$ with respect to $y$. According to (20), (21), we obtain the original equation in the form

$$
\begin{array}{r}
\frac{\partial\left(d+d_{t}\right)}{\partial y}=-\frac{1}{3}\left(u \frac{\partial u}{\partial y}+v \frac{\partial v}{\partial y}+w \frac{\partial w}{\partial y}\right)+\frac{1}{3}\left(-\frac{\partial^{3} \Psi_{10}}{\partial x^{2} \partial y}-\frac{\partial^{3} \Psi_{10}}{\partial y^{3}}+\frac{\partial^{3} \Psi_{11}}{\partial x^{2} \partial y}+\frac{\partial^{3} \Psi_{11}}{\partial y \partial z^{2}}-\right. \\
\left.-\frac{\partial^{3} \Psi_{12}}{\partial y^{3}}-\frac{\partial^{3} \Psi_{12}}{\partial y \partial z^{2}}-\frac{\partial^{3} \Psi_{13}}{\partial x \partial y^{2}}+\frac{\partial^{3} \Psi_{14}}{\partial x \partial y \partial z}-\frac{\partial^{3} \Psi_{15}}{\partial y^{2} \partial z}\right)+\frac{1}{3} \frac{\partial}{\partial t}\left(\frac{\partial^{2} \Psi_{2}}{\partial x \partial y}-\frac{\partial^{2} \Psi_{1}}{\partial x \partial y}+\frac{\partial^{2} \Psi_{4}}{\partial y^{2}}-\right. \\
\left.-\frac{\partial^{2} \Psi_{3}}{\partial y^{2}}+\frac{\partial^{2} \Psi_{6}}{\partial y \partial z}-\frac{\partial^{2} \Psi_{5}}{\partial y \partial z}\right)
\end{array}
$$

Let us simplify the term with third derivatives on the right-hand side. Using (11-18), we find $-\frac{1}{3} \frac{\partial}{\partial y}$ of (11), $\frac{1}{3} \frac{\partial}{\partial y}$ of (12), $\frac{\partial}{\partial x}$ of (13) and $\frac{\partial}{\partial z}$ of (15). Then we obtain an expression for the needed term

$$
\begin{aligned}
& \frac{1}{3}\left(-\frac{\partial^{3} \Psi_{10}}{\partial x^{2} \partial y}-\frac{\partial^{3} \Psi_{10}}{\partial y^{3}}+\frac{\partial^{3} \Psi_{11}}{\partial x^{2} \partial y}+\frac{\partial^{3} \Psi_{11}}{\partial y \partial z^{2}}-\frac{\partial^{3} \Psi_{12}}{\partial y^{3}}-\frac{\partial^{3} \Psi_{12}}{\partial y \partial z^{2}}-\frac{\partial^{3} \Psi_{13}}{\partial x \partial y^{2}}+\frac{\partial^{3} \Psi_{14}}{\partial x \partial y \partial z}-\frac{\partial^{3} \Psi_{15}}{\partial y^{2} \partial z}\right)= \\
& =\frac{4}{3} v \frac{\partial v}{\partial y}-\frac{2}{3} u \frac{\partial u}{\partial y}-\frac{2}{3} w \frac{\partial w}{\partial y}+v \frac{\partial u}{\partial x}+u \frac{\partial v}{\partial x}+w \frac{\partial v}{\partial z}+v \frac{\partial w}{\partial z}+\frac{1}{R e}\left(-\frac{4}{3} \frac{\partial^{2} v}{\partial y^{2}}-\frac{1}{3} \frac{\partial^{2} u}{\partial x \partial y}-\frac{1}{3} \frac{\partial^{2} w}{\partial y \partial z}-\right. \\
& \left.-\frac{\partial^{2} v}{\partial x^{2}}-\frac{\partial^{2} v}{\partial z^{2}}\right)+\frac{\partial}{\partial t}\left(-\frac{1}{6} \frac{\partial^{2} \Psi_{1}}{\partial x \partial y}-\frac{1}{3} \frac{\partial^{2} \Psi_{2}}{\partial x \partial y}+\frac{1}{2} \frac{\partial^{2} \Psi_{3}}{\partial x^{2}}+\frac{1}{3} \frac{\partial^{2} \Psi_{3}}{\partial y^{2}}-\frac{1}{3} \frac{\partial^{2} \Psi_{4}}{\partial y^{2}}-\frac{1}{2} \frac{\partial^{2} \Psi_{4}}{\partial z^{2}}+\frac{1}{3} \frac{\partial^{2} \Psi_{5}}{\partial y \partial z}+\right.
\end{aligned}
$$




$$
\left.+\frac{1}{6} \frac{\partial^{2} \Psi_{6}}{\partial y \partial z}-\frac{1}{2} \frac{\partial^{2} \Psi_{7}}{\partial x \partial z}+\frac{1}{2} \frac{\partial^{2} \Psi_{9}}{\partial x \partial z}\right)+\frac{1}{2} \frac{\partial^{2} \beta_{2}}{\partial x \partial t}+\frac{1}{2} \frac{\partial^{2} \beta_{3}}{\partial z \partial t}+\frac{\partial \beta_{4}}{\partial y}+\frac{\partial \gamma_{4}}{\partial y}
$$

Taking into account (30), we transform (29) as

$$
\begin{gathered}
\frac{\partial\left(d+d_{t}\right)}{\partial y}=\frac{1}{R e}\left(-\Delta v-\frac{1}{3} \frac{\partial}{\partial y}\left(\frac{\partial u}{\partial x}+\frac{\partial v}{\partial y}+\frac{\partial w}{\partial z}\right)\right)+v\left(\frac{\partial u}{\partial x}+\frac{\partial v}{\partial y}+\frac{\partial w}{\partial z}\right)+u\left(\frac{\partial v}{\partial x}-\frac{\partial u}{\partial y}\right)+ \\
+w\left(\frac{\partial v}{\partial z}-\frac{\partial w}{\partial y}\right)+\frac{1}{2} \frac{\partial}{\partial t}\left(-\frac{\partial^{2} \Psi_{1}}{\partial x \partial y}+\frac{\partial^{2} \Psi_{3}}{\partial x^{2}}-\frac{\partial^{2} \Psi_{4}}{\partial z^{2}}+\frac{\partial^{2} \Psi_{6}}{\partial y \partial z}-\frac{\partial^{2} \Psi_{7}}{\partial x \partial z}+\frac{\partial^{2} \Psi_{9}}{\partial x \partial z}\right)+ \\
+\frac{1}{2} \frac{\partial^{2} \beta_{2}}{\partial x \partial t}+\frac{1}{2} \frac{\partial^{2} \beta_{3}}{\partial z \partial t}+\frac{\partial \alpha_{4}}{\partial y}+\frac{\partial \gamma_{4}}{\partial y}
\end{gathered}
$$

Using (23) together with continuity equation (4), we find that first group of terms on the right-hand side of (31) vanishes. Let us note that the second group of terms is also vanishes as a consequence of equation (4) and conditions (24). If we use (17) and (19) then all the remaining terms on the right-hand side are reduced to $\frac{\partial v}{\partial t}+\frac{\partial \alpha_{4}}{\partial y}+\frac{\partial \gamma_{4}}{\partial y}$.

As a result relation (31) takes the form

$$
\frac{\partial\left(d+d_{t}\right)}{\partial y}=\frac{\partial v}{\partial t}+\frac{\partial \alpha_{4}}{\partial y}+\frac{\partial \gamma_{4}}{\partial y}
$$

2.1.3. Let us take the first derivative of $d+d_{t}$ with respect to $z$. Using (20), (21), we obtain

$$
\begin{array}{r}
\frac{\partial\left(d+d_{t}\right)}{\partial z}=-\frac{1}{3}\left(u \frac{\partial u}{\partial z}+v \frac{\partial v}{\partial z}+w \frac{\partial w}{\partial z}\right)+\frac{1}{3}\left(-\frac{\partial^{3} \Psi_{10}}{\partial x^{2} \partial z}-\frac{\partial^{3} \Psi_{10}}{\partial y^{2} \partial z}+\frac{\partial^{3} \Psi_{11}}{\partial x^{2} \partial z}+\frac{\partial^{3} \Psi_{11}}{\partial z^{3}}-\right. \\
\left.-\frac{\partial^{3} \Psi_{12}}{\partial y^{2} \partial z}-\frac{\partial^{3} \Psi_{12}}{\partial z^{3}}-\frac{\partial^{3} \Psi_{13}}{\partial x \partial y \partial z}+\frac{\partial^{3} \Psi_{14}}{\partial x \partial z^{2}}-\frac{\partial^{3} \Psi_{15}}{\partial y \partial z^{2}}\right)+ \\
+\frac{1}{3} \frac{\partial}{\partial t}\left(\frac{\partial^{2} \Psi_{2}}{\partial x \partial z}-\frac{\partial^{2} \Psi_{1}}{\partial x \partial z}+\frac{\partial^{2} \Psi_{4}}{\partial y \partial z}-\right. \\
\left.-\frac{\partial^{2} \Psi_{3}}{\partial y \partial z}+\frac{\partial^{2} \Psi_{6}}{\partial z^{2}}-\frac{\partial^{2} \Psi_{5}}{\partial z^{2}}\right)
\end{array}
$$

Using (11-15), a group of terms with third derivatives on the right-hand side of (33) can be converted. Let us find $-\frac{1}{3} \frac{\partial}{\partial z}$ of (11), $-\frac{2}{3} \frac{\partial}{\partial z}$ of (12), $\frac{\partial}{\partial x}$ of (14), $\frac{\partial}{\partial y}$ of (15). Then we obtain an expression for the needed term with third derivatives in the form

$$
\begin{gathered}
\frac{1}{3}\left(-\frac{\partial^{3} \Psi_{10}}{\partial x^{2} \partial z}-\frac{\partial^{3} \Psi_{10}}{\partial y^{2} \partial z}+\frac{\partial^{3} \Psi_{11}}{\partial x^{2} \partial z}+\frac{\partial^{3} \Psi_{11}}{\partial z^{3}}-\frac{\partial^{3} \Psi_{12}}{\partial z^{3}}-\frac{\partial^{3} \Psi_{12}}{\partial y \partial z^{2}}-\frac{\partial^{3} \Psi_{13}}{\partial x \partial y \partial z}+\frac{\partial^{3} \Psi_{14}}{\partial x \partial z^{2}}-\frac{\partial^{3} \Psi_{15}}{\partial y \partial z^{2}}\right)= \\
=-\frac{2}{3} u \frac{\partial u}{\partial z}-\frac{2}{3} v \frac{\partial v}{\partial z}+\frac{4}{3} w \frac{\partial w}{\partial z}+w \frac{\partial u}{\partial x}+u \frac{\partial w}{\partial x}+w \frac{\partial v}{\partial y}+v \frac{\partial w}{\partial y}+\frac{1}{R e}\left(-\frac{1}{3} \frac{\partial^{2} v}{\partial y \partial z}-\frac{1}{3} \frac{\partial^{2} u}{\partial x \partial z}-\frac{4}{3} \frac{\partial^{2} w}{\partial z^{2}}-\right. \\
\left.-\frac{\partial^{2} w}{\partial x^{2}}-\frac{\partial^{2} w}{\partial y^{2}}\right)+\frac{\partial}{\partial t}\left(\frac{1}{3} \frac{\partial^{2} \Psi_{1}}{\partial x \partial z}+\frac{1}{6} \frac{\partial^{2} \Psi_{2}}{\partial x \partial z}+\frac{1}{3} \frac{\partial^{2} \Psi_{3}}{\partial y \partial z}+\frac{1}{6} \frac{\partial^{2} \Psi_{4}}{\partial y \partial z}+\frac{1}{3} \frac{\partial^{2} \Psi_{5}}{\partial z^{2}}+\frac{1}{2} \frac{\partial^{2} \Psi_{5}}{\partial x^{2}}-\frac{1}{3} \frac{\partial^{2} \Psi_{6}}{\partial z^{2}}-\right. \\
\left.-\frac{1}{2} \frac{\partial^{2} \Psi_{6}}{\partial y^{2}}-\frac{1}{2} \frac{\partial^{2} \Psi_{8}}{\partial x \partial y}-\frac{1}{2} \frac{\partial^{2} \Psi_{9}}{\partial x \partial y}\right)+\frac{1}{2} \frac{\partial^{2} \gamma_{2}}{\partial y \partial t}+\frac{1}{2} \frac{\partial^{2} \gamma_{3}}{\partial x \partial t}+\frac{\partial \alpha_{4}}{\partial z}+\frac{\partial \beta_{4}}{\partial z} .
\end{gathered}
$$

Using (34), we transform (33) and obtain 


$$
\begin{gathered}
\frac{\partial\left(d+d_{t}\right)}{\partial z}=\frac{1}{R e}\left(-\Delta w-\frac{1}{3} \frac{\partial}{\partial z}\left(\frac{\partial u}{\partial x}+\frac{\partial v}{\partial y}+\frac{\partial w}{\partial z}\right)\right) \\
+v\left(\frac{\partial w}{\partial y}-\frac{\partial v}{\partial z}\right)+\frac{1}{2} \frac{\partial}{\partial t}\left(\frac{\partial^{2} \Psi_{2}}{\partial x \partial z}+\frac{\partial^{2} \Psi_{4}}{\partial y \partial z}+\frac{\partial^{2} \Psi_{5}}{\partial x^{2}}-\frac{\partial^{2} \Psi_{6}}{\partial y^{2}}-\frac{\partial^{2} \Psi_{8}}{\partial x \partial y}-\frac{\partial^{2} \Psi_{9}}{\partial x \partial y}\right)+ \\
+\frac{1}{2} \frac{\partial^{2} \gamma_{2}}{\partial y \partial t}+\frac{1}{2} \frac{\partial^{2} \gamma_{3}}{\partial x \partial t}+\frac{\partial \alpha_{4}}{\partial z}+\frac{\partial \beta_{4}}{\partial z}
\end{gathered}
$$

We use continuity equation (4) together with (23) and find that the terms proportional to $\frac{1}{R}$

$\frac{1}{R e}$ vanishes. The second group of terms is also vanishes due to equation (4) and conditions (24). Using equations (18) and (19), we obtain that remaining terms on the right-hand side of (35) are reduced to $\frac{\partial w}{\partial t}+\frac{\partial \alpha_{4}}{\partial z}+\frac{\partial \beta_{4}}{\partial z}$.

Finally, relation (35) takes the form

$$
\frac{\partial\left(d+d_{t}\right)}{\partial z}=\frac{\partial w}{\partial t}+\frac{\partial \alpha_{4}}{\partial z}+\frac{\partial \beta_{4}}{\partial z} .
$$

2.1.4. The first derivatives of $d+d_{t}$ with respect to $x, y, z$ are defined by (28), (32), (36). These relations can be written in a more convenient form if we add in the right-hand sides $\frac{\partial \alpha_{4}}{\partial x}$, $\frac{\partial \beta_{4}}{\partial y}, \frac{\partial \gamma_{4}}{\partial z}$, respectively. Each of these quantities is equal to zero under condition of (19), so relations (28), (32), (36) are valid. Using (22) and changing the order of differentiation with respect to time and coordinates in the second mixed derivatives, we find that relations (28), (32), (36) take the form

$$
\begin{aligned}
& \frac{\partial\left(d+d_{t}\right)}{\partial x}=\frac{\partial}{\partial x}\left(\frac{\partial \varphi}{\partial t}+\alpha_{4}+\beta_{4}+\gamma_{4}\right) \\
& \frac{\partial\left(d+d_{t}\right)}{\partial y}=\frac{\partial}{\partial y}\left(\frac{\partial \varphi}{\partial t}+\alpha_{4}+\beta_{4}+\gamma_{4}\right) \\
& \frac{\partial\left(d+d_{t}\right)}{\partial z}=\frac{\partial}{\partial z}\left(\frac{\partial \varphi}{\partial t}+\alpha_{4}+\beta_{4}+\gamma_{4}\right)
\end{aligned}
$$

Quantities $\left(d+d_{t}\right)$ and $\left(\frac{\partial \varphi}{\partial t}+\alpha_{4}+\beta_{4}+\gamma_{4}\right)$ have the same derivatives with respect to coordinates. Hence, they may differ only by an additive arbitrary function of time $-f(t)$. Then we have

$$
d+d_{t}=\frac{\partial \varphi}{\partial t}+\alpha_{4}+\beta_{4}+\gamma_{4}-f(t)
$$

Substituting (38) into (10), we obtain relation that is equivalent to (5)

$$
p+\Phi+\frac{U^{2}}{2}+\frac{\partial \varphi}{\partial t}-f(t)=0
$$

Thus, the Lagrange-Cauchy integral (5) is a special case of root integral (10-18).

The proof of Theorem 2.1 is complete.

\section{Integral of Bernoulli and Euler - Bernoulli as special cases of the root integral}

The following theorems are valid. 
Theorem 3.1 The Bernoulli integral (6) is a special case of the root integral (10-18).

Proof. The derivation of the Bernoulli integral is based on three assumptions [1-4]. We assume that medium is ideal, motion is steady-state, and characteristic point is selected along a stream line. We prove that under these assumptions the Bernoulli integral (6) is a consequence of $(10-18)$.

According to first two assumptions, we have $\frac{\partial}{\partial t}=0$ and $\frac{1}{R e}=0$. The latter implies that $d_{t}=0$ and relation (10) takes the form

$$
p+\Phi+\frac{U^{2}}{2}+d=\alpha_{4}+\beta_{4}+\gamma_{4}
$$

The left-hand side of this equation differs from the Bernoulli trinomial by the value $d$. Let us find the derivatives of $d$ with respect to spatial coordinates. To do this one can use relations (27), (31), (35). Note that these relations are simplified under given assumptions and take the form

$$
\begin{aligned}
& \frac{\partial d}{\partial x}=u\left(\frac{\partial u}{\partial x}+\frac{\partial v}{\partial y}+\frac{\partial w}{\partial z}\right)+v\left(\frac{\partial u}{\partial y}-\frac{\partial v}{\partial x}\right)+w\left(\frac{\partial u}{\partial z}-\frac{\partial w}{\partial x}\right)+\frac{\partial \beta_{4}}{\partial x}+\frac{\partial \gamma_{4}}{\partial x} \\
& \frac{\partial d}{\partial y}=v\left(\frac{\partial u}{\partial x}+\frac{\partial v}{\partial y}+\frac{\partial w}{\partial z}\right)+u\left(\frac{\partial v}{\partial x}-\frac{\partial u}{\partial y}\right)+w\left(\frac{\partial v}{\partial z}-\frac{\partial w}{\partial y}\right)+\frac{\partial \alpha_{4}}{\partial y}+\frac{\partial \gamma_{4}}{\partial y} \\
& \frac{\partial d}{\partial z}=w\left(\frac{\partial u}{\partial x}+\frac{\partial v}{\partial y}+\frac{\partial w}{\partial z}\right)+u\left(\frac{\partial w}{\partial x}-\frac{\partial u}{\partial z}\right)+v\left(\frac{\partial w}{\partial y}-\frac{\partial v}{\partial z}\right)+\frac{\partial \alpha_{4}}{\partial z}+\frac{\partial \beta_{4}}{\partial z}
\end{aligned}
$$

First term on the right-hand side vanishes due to continuity equation (4). To do further simplifications the following steps are performed. As it was done in Section 2.1.4, we add in right-hand side (40) the terms $\frac{\partial \alpha_{4}}{\partial x}, \frac{\partial \beta_{4}}{\partial y}, \frac{\partial \gamma_{4}}{\partial z}$, respectively. Each of these quantities is equal to zero so relation (40) stays valid. We left non-linear terms on the right-hand sides, and rearrange all other terms to the left-hand sides. Then (40) takes the form

$$
\begin{aligned}
& \frac{\partial\left(d-\alpha_{4}-\beta_{4}-\gamma_{4}\right)}{\partial x}=v\left(\frac{\partial u}{\partial y}-\frac{\partial v}{\partial x}\right)+w\left(\frac{\partial u}{\partial z}-\frac{\partial w}{\partial x}\right) \\
& \frac{\partial\left(d-\alpha_{4}-\beta_{4}-\gamma_{4}\right)}{\partial y}=u\left(\frac{\partial v}{\partial x}-\frac{\partial u}{\partial y}\right)+w\left(\frac{\partial v}{\partial z}-\frac{\partial w}{\partial y}\right) \\
& \frac{\partial\left(d-\alpha_{4}-\beta_{4}-\gamma_{4}\right)}{\partial z}=u\left(\frac{\partial w}{\partial x}-\frac{\partial u}{\partial z}\right)+v\left(\frac{\partial w}{\partial y}-\frac{\partial v}{\partial z}\right)
\end{aligned}
$$

The right-hand side has obvious symmetry. Let us multiply the first relation (41) by $u$, the second by $v$, the third by $w$ and add up the resulting relations. We obtain that all terms of the right-hand sides cancel each other and the resulting relation takes the form

$$
u \frac{\partial\left(d-\alpha_{4}-\beta_{4}-\gamma_{4}\right)}{\partial x}+v \frac{\partial\left(d-\alpha_{4}-\beta_{4}-\gamma_{4}\right)}{\partial y}+w \frac{\partial\left(d-\alpha_{4}-\beta_{4}-\gamma_{4}\right)}{\partial z}=0 .
$$

Relation (42) can be written in vector form as

$$
\vec{U} \cdot \vec{\nabla}\left(d-\alpha_{4}-\beta_{4}-\gamma_{4}\right)=0
$$

The latter implies that the value of $\left(d-\alpha_{4}-\beta_{4}-\gamma_{4}\right)$ does not change on direction given by the velocity vector $\vec{U}(u, v, w)$. It means that it does not change along a stream line. So, along the stream line the following equality hold 


$$
\left(d-\alpha_{4}-\beta_{4}-\gamma_{4}\right)=-C_{s l},
$$

where $C_{s l}$ is a constant value corresponded to the selected stream line.

The value $d$ determined by (44) is $d=\alpha_{4}+\beta_{4}+\gamma_{4}-C_{s l}$. Substituting this in (39), we obtain the following relation along a stream line

$$
p+\Phi+\frac{U^{2}}{2}-C_{s l}=0 .
$$

This is equivalent to the Bernoulli integral (6). Thus, the Bernoulli integral (6) is a special case of the root integral (10-18).

Proof of Theorem 3.1 is complete.

Theorem 3.2 The Euler-Bernoulli integral (8) is a special case of the root integral (10-18).

Proof. The derivation of the Euler-Bernoulli integral is base on three assumptions [1]. We assume that medium is ideal, motion is steady-state and condition (7) is satisfied. We show that under these assumptions (8) follows from (10-18).

Assumptions of ideality of a medium and the steady-state nature of motion coincides with the assumptions of Section 3.1. It means that we can use some relations of the previous section, in particular, relations (39) and (41). However, subject to (7) each of the right-hand sides of (41) vanishes. Thus, in our case we have

$$
\frac{\partial\left(d-\alpha_{4}-\beta_{4}-\gamma_{4}\right)}{\partial x}=\frac{\partial\left(d-\alpha_{4}-\beta_{4}-\gamma_{4}\right)}{\partial y}=\frac{\partial\left(d-\alpha_{4}-\beta_{4}-\gamma_{4}\right)}{\partial z}=0 .
$$

This implies that the value of $\left(d-\alpha_{4}-\beta_{4}-\gamma_{4}\right)$ is independent of spatial coordinates and it is equal to constant:

$$
\left(d-\alpha_{4}-\beta_{4}-\gamma_{4}\right)=-C .
$$

Substituting (47) into (39) we obtain

$$
p+\Phi+\frac{U^{2}}{2}-C=0 .
$$

The latter is equivalent to (8). Thus, the Euler-Bernoulli integral is a special case of integral $(10-18)$.

Proof of Theorem 3.2 is complete.

\section{Systematization of integrals of motion for an incompressible fluid flow}

Thus, integrals (5), (6), (8) are special cases of the general integral represented by nine relations (10-18). We say that (10-18) is integral " A". The significance of this integral is that it can be the basis for other new integrals. Let us point out few of these cases.

First we consider the integral for motion of an ideal incompressible fluid. We call it the integral "I". It is derived from "A" with the use of condition $\frac{1}{R e}=0$. In this case changes occur only in five relations (11-15). The resulting integral "I" presented by nine ratios as well as the general integral " A".

For the steady-state motion of incompressible viscous fluid occurs integral which we call the integral "S". It is derived from "A" with the use of condition $\frac{\partial}{\partial t}=0$. In this case three 
relations (16-18) are replaced with continuity equation (4). For this case integral is presented by six relationships [9]. Let us note that number of associated unknowns $\Psi_{i}$ is reduced to six.

Another possible integral in this family corresponds to the case of steady state-motion of an ideal incompressible fluid. We call it the "IS " integral. There are two different ways to derive this integral. First one is to use the " $\mathrm{S}$ " integral with condition $\frac{1}{R e}=0$. The second one is to use the "I" integral with with condition $\frac{\partial}{\partial t}=0$. The "IS " integral is represented by six relations. When comparing it with the integral " $\mathrm{S}$ " changes take place only in the last five relations. Terms proportional to $\frac{1}{R e}=0$ vanishes.

There are other less obvious integrals that are generated by the " $\mathrm{A}$ " integral. For this we consider the Lagrange-Cauchy integral (5). It is a special case of the " $\mathrm{A}$ " integral. As follows from the above considerations, integral (5) is the result of changes in relation (10). However, remaining relations (11-18) also remain valid. The combination of these eight interconnected relations can be regarded as a new integral. This new integral we call the "L2", integral while integral (5) is called the "L1" integral. So, integral "L2" obtained under the same assumptions that integral "L1" and it contains eight relations.

A similar situation occurs with integrals (6) and (8). Both integrals correspond to the case of steady-state motion of an ideal fluid flow. Both integrals can be obtained from the "IS ", integral represented by six relations. It follows from Sections 3.1 and 3.2 that both integrals are the result of conversions of relation (10). However, the remaining five relations are also valid. If we denote the Bernoulli integral (6) as the "B1" integral and the Euler-Bernoulli integral (8) as the" E1" integral then " B2 " and " E2 " integrals naturally arises. Each of these new integrals represented by five relations. Moreover, the " B2 " integral corresponds to the assumptions of the Bernoulli integral and the " E2 " integral corresponds to the assumptions of the Euler-Bernoulli integral.

Thus, the " A" integral generates, in addition to known integrals, six new integrals. These integrals are "S ", "I", " IS ", " B2 ", " E2 ", " L2 ".

To show various relationships between integrals more clearly, they are conveniently presented in a form of tree. It follows from considerations given above that the "A " integral should be placed at the base of the tree. All other integrals are special cases of the "A" integral. They constitute two main branches. The right-hand branch contains integrals of motion for viscous fluid "S ", "L1", "L2". The left-hand branch contains integrals for the particular cases of an ideal fluid "I", " IS ", "B1", "B2 ", "E1", " E2 ". All of these ten integrals form a tree of integrals of motion for an incompressible fluid flow. The tree is shown in Fig. 1.

\section{Conclusion}

All integrals of incompressible fluid flow, both known and obtained in the paper, are presented in the form of a tree. The " A" integral is at the base of the tree as the general one. The " A" integral for this reason is fully justified the name the first integral of motion for an incompressible fluid flow. All other integrals are special cases of the " $\mathrm{A}$ " integral. It is possible that new not yet considered integrals may appear in this tree in the future.

Each new integral contains relationships between the governing values. These relations can simplify the solution of hydrodynamical problems. This approach has been already implemented, and solutions of hydrodynamical problems were based on "A", " S " and " IS " integrals[12-16]. 


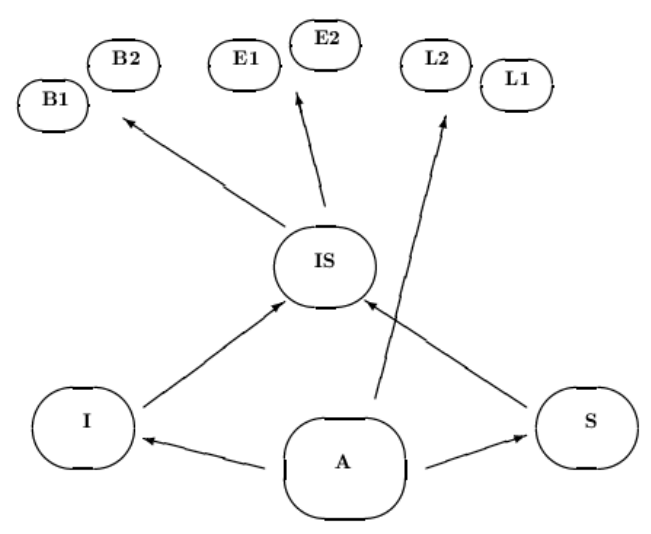

Fig. 1. Tree of integrals for an incompressible fluid flow

\section{References}

[1] L.I.Sedov, Continuum mechanics, Part 2, Moscow, Nauka, 1970 (in Russian).

[2] L.G.Loitsaynskiy, Mechanics of Fluid and Gas, Moscow, Nauka, 1987 (in Russian).

[3] N.E.Kochin, I.A.Kibel, N.V.Rose, Theoretical hydromechanics, Part 1, Moscow, Fismatlit, 1963 (in Russian).

[4] S.V.Vallander, Lectures on hydromechanics, Leningrad Gos. Univ., Leningrad, 1978 (in Russian).

[5] O.A.Ladizhenskaya, The Mathematical Theory of Viscous Incompressible Fluid, Gordon and Breach, New York, 1969.

[6] C.L.Fefferman, Existence and Smoothness of the Navier-Stokes Equation, Preprint, Princeton Univ., Math. Dept., Princeton, NJ, USA, 2000.

[7] A.V.Koptev, Integrals of the Navier-Stokes Equations, Trudy Sredne-volzhskogo Matematicheskogo Obshchestva, Saransk, 6(1)(2004), no. 1, 215-225 (in Russian).

[8] A.V.Koptev, First integral and Ways of Further Integration of Navier-Stokes Equations, Izv. Ross. gos. pedagog. univ. im. Gertsena, St-Petersburg, 147 (2012), 7-17 (in Russian).

[9] A.V.Koptev, First integral of motion for an incompressible fluid flow, Proc. of 11-th AllRussian Congress on fundamental problems of theoretical and applied mechanics, Privolzhskiy Federal Univ., Kazan, 2015, 1957-1959 (in Russian).

[10] A.V.Koptev, Nonlinear Effects in Poiseuille Problem, Journal of Siberian Federal University. Math. and Phys., 6(2013), no. 3, 308-314.

[11] V.M.Zhuravlev, A new representation of two-dimensional equations of the dynamics of an incompressible fluid, Prikladnaya matematika i mekhanika, 58(1994), no. 6, 61-67 (in Russian).

[12] A.D.Polynin, A.I.Zhurov, Parametically defined nonlinear differential equations and their solutions, Applied Math. Letters, 55(2016), 72-80. 
[13] A.V.Koptev, Generator of Solution of 2D Navier-Stokes Equations, Journal of Siberian Federal University, Math. and Phys., 7(2014), no. 3, 324-330.

[14] A.V.Koptev, The Solution of Initial and Boundarry Value Problem for 3D Navier-Stokes Equations and its Features, Izv. Ross. gos. pedagog. univ. im. Gertsena, St-Petersburg, 165(2014), 7-18 (in Russian).

[15] A.V.Koptev, Dynamic Responce of an Underwater Pipeline on the Sea Currents, Vestnik gos. univ. morskogo i rechnogo flota im. adm. S.O. Makarova, St-Petersburg, 26(2014), no. 4, 107-114 (in Russian).

[16] A.V.Koptev, Theoretical Research of the Flow Around the Cylinder of an Ideal Incompressible Medium in the Presence of a Shielding Effect, Vestnik gos. univ. morskogo i rechnogo flota im. adm. S.O. Makarova, St-Petersburg, 36(2016), no. 2, 127-137 (in Russian).

\section{Систематизация и анализ интегралов движения несжимаемой жидкости}

Александр В. Коптев

Гос. университет морского и речного флота им. адмирала С. О. Макарова Двинская, 5/7, Санкт-Петербург, 198035

Россия

В работе рассмотрены и проанализированы интеграль движения несжимаемой среды, как известные, так и новые, полученные автором. Приводится доказательство того, что известные интегралы Лагранжа - Коши, Бернулли и Эйлера - Бернулли есть частные случаи нового более общего интеграла. Показано, что множество всех интегралов движения несжимаемой жидкости образует логическую чепочку, которую можно представить в виде дерева.

Ключевые слова: несжимаемая жидкость, движение, уравнения Навъе-Стокса, уравнения Эйлера, частная производная, корневой интеграл, псевдофункиия тока, потенииал, дерево. 